\title{
Correction: PBK phosphorylates MSL1 to elicit epigenetic modulation of CD276 in nasopharyngeal carcinoma
}

Meng-Yao Wang, Bin Qi, Fang Wang, Zhi-Rui Lin, Ming-Yi Li, Wen-Jing Yin, Yan-Yi Zhu, Lu He, Yi Yu, Fang Yang, Jin-Quan Liu and Dong-Ping Chen (D)

Correction to: Oncogenesis

https://doi.org/10.1038/s41389-020-00293-9

published online 5 January 2021

The information that these authors contributed equally: Meng-Yao Wang, Bin Qi, Fang Wang, should include in the author information page.
The original article has been corrected.

Published online: 30 April 2021 